\title{
Miscibility and Crystallization Behavior of Crystalline/Crystalline Polymer Blends. Poly(ester carbonate)/Poly(L-lactic acid)
}

\author{
Satomi Hirano, Yuko Nishikawa, Yasuhiko TeradA, \\ Takayuki IKEHARA, and Toshio NISHI \\ Department of Applied Physics, School of Engineering, The University of Tokyo, 7-3-1 Hongo, \\ Bunkyo-ku, Tokyo 113-8656, Japan
}

(Received October 12, 2001; Accepted December 17, 2001)

\begin{abstract}
KEY WORDS Poly(butylene succinate-co-butylene carbonate) / Poly(L-lactic acid) / Crystalline Polymer Blends / Interpenetrated Spherulites /
\end{abstract}

The numbers of the studies on miscible pairs of crystalline polymers with different chemical structures are still restricted compared with those with similar chemical structures. ${ }^{1-10}$ The differences of the melting temperatures $T_{\mathrm{m}}$ of the components are usually $100^{\circ} \mathrm{C}$ in the former systems. In these cases, the higher- $T_{\mathrm{m}}$ component crystallizes first in the crystallization process and the other component then crystallizes in spatially constrained amorphous regions inside the spherulites.

In contrast, when $T_{\mathrm{m}} \mathrm{s}$ of both components are close enough to each other, the blends exhibit simultaneous spherulitic growth. The spherulites in poly(butylene succinate)/poly(vinylidene chloride-co-vinyl chloride) (PBSU/PVDCVC) blends, whose $T_{\mathrm{m}}$ difference is about $35^{\circ} \mathrm{C}$, grow simultaneously. These blends were found to show interpenetrated spherulites (IPS). ${ }^{11,12}$ They are characterized by the penetration of PBSU lamellae into PVDCVC spherulites since the lamellar population density in a spherulite is larger for PBSU than for PVDCVC. ${ }^{13,14}$ They are expected to have various unique physical characteristics.

The reports on the blend systems that show IPS are very few. This is partly because miscible crystalline polymers rarely have close $T_{\mathrm{m}} \mathrm{s}$. Our group has been searching polymer blends that show IPS.

Poly(butylene succinate-co-butylene carbonate) (PEC) and poly(L-lactic acid) (PLLA) are polyesters whose $T_{\mathrm{m}} \mathrm{s}$ are close to each other $\left(106^{\circ} \mathrm{C}\right.$ and $140^{\circ} \mathrm{C}$, respectively). If they are miscible, they possibly crystallize simultaneously and show IPS. Furthermore, since both PEC and PLLA are biodegradable, PEC/PLLA blends can be ecological crystalline polymer blends.

The purposes of this paper are to show miscibility of PEC/PLLA by differential scanning calorimetry
(DSC), to study the crystallization behavior by polarizing optical microscopy (POM), and to investigate the possibility of IPS.

\section{EXPERIMENTAL}

$\operatorname{PEC}\left(M_{\mathrm{w}} \approx 10^{5}, T_{\mathrm{m}}=106^{\circ} \mathrm{C}, T_{\mathrm{g}}=-26^{\circ} \mathrm{C}\right)$ provided by Mitsubishi Gas Chemical Company Inc. is a random copolymer of butylene succinate and butylene carbonate. PLLA $\left(M_{\mathrm{w}}=2000, T_{\mathrm{m}}=140^{\circ} \mathrm{C}\right.$, $T_{\mathrm{g}}=40^{\circ} \mathrm{C}$ ) was purchased from Polysciences Inc. PEC/PLLA blends were prepared with mutual solvent chloroform at room temperature. The solution was cast on glass plates and then the solvent was removed at room temperature to make thin films for POM observation. For DSC, the solution was dried for a week and then in a vacuum for three days to remove the solvent from the bulky samples.

The glass transition temperature $T_{\mathrm{g}}$ was determined by DSC (TA Instruments DSC 2910). Samples were heated to $170^{\circ} \mathrm{C}$ for $5 \mathrm{~min}$, quenched to $-100^{\circ} \mathrm{C}$ using liquid nitrogen, kept for $5 \mathrm{~min}$, and heated at $10^{\circ} \mathrm{C} \mathrm{min}^{-1}$. The value of $T_{\mathrm{g}}$ was determined as the temperature of the half-height of the step transition.

To study the morphology of the blends and the radial spherulitic growth rates $G$ of PEC and PLLA, we used POM (Olympus BHP-P) equipped with a first order retardation plate and a temperature controller (Linkam TH-600). Samples were premelted at $170^{\circ} \mathrm{C}$ for $3 \mathrm{~min}$ and then quenched to the crystallization temperature $T_{\mathrm{c}}$.

\section{RESULTS AND DISCUSSION}

To confirm the miscibility of the PEC/PLLA blends, we measured DSC endotherms (Figure 1). All the 


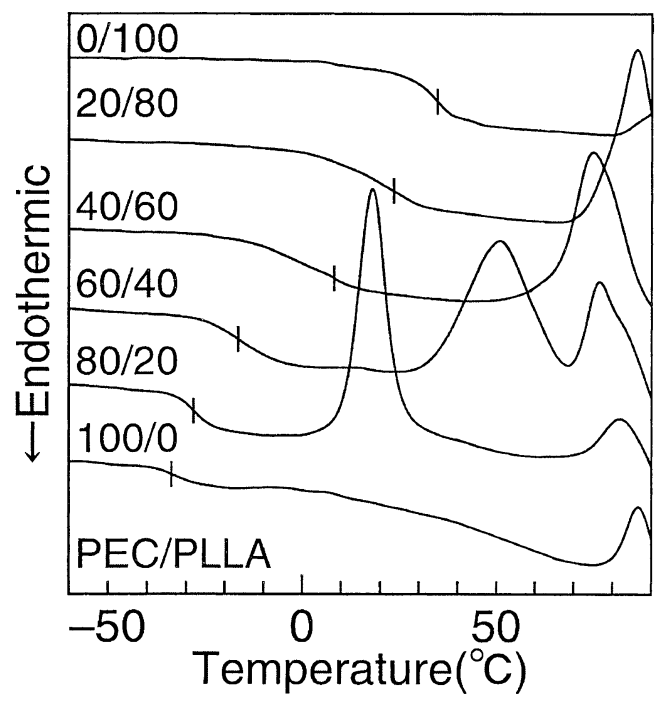

Figure 1. DSC endotherms of PEC/PLLA blends. The values of $T_{\mathrm{g}}$ are indicated by the vertical lines.

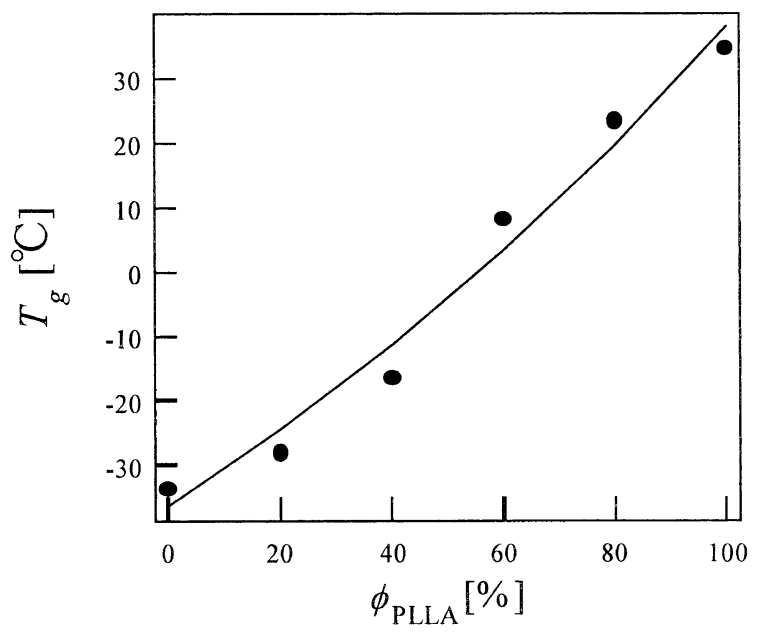

Figure 2. Dependence of $T_{\mathrm{g}}$ of the PEC/PLLA blends on the content of PLLA. The solid line represents the Fox equation fitted to the $T_{\mathrm{g}}$ values.

blends showed a single $T_{\mathrm{g}}$. Peaks at the right hand side of $T_{\mathrm{g}} \mathrm{s}$ are re-crystallization peaks. The experimental values of $T_{\mathrm{g}}$ are plotted against the PLLA content $\phi_{\text {PLLA }}$ in Figure 2. The values of $T_{\mathrm{g}}$ fitted to the Fox equation. ${ }^{15}$ These results indicate miscibility of the blends.

POM observations showed that PEC spherulites filled the whole area and that no PLLA spherulites grew for $\phi_{\text {PLLA }} \lesssim 0.3$ and $T_{\mathrm{c}} \lesssim 60^{\circ} \mathrm{C}$ (regime i). Figure 3 is a typical POM micrograph in regime $\mathrm{i}$.

On the contrary, only PLLA spherulites grew $0.2 \lesssim \phi_{\mathrm{PLLA}}$ and $90^{\circ} \mathrm{C} \lesssim T_{\mathrm{c}}$ (regime iv). Figure 4 is a typical POM image of regime iv.

Under intermediate conditions $\left(0.1 \lesssim \phi_{\text {PLLA }} \lesssim 0.6\right.$ and $60^{\circ} \mathrm{C} \lesssim T_{\mathrm{c}} \lesssim 90^{\circ} \mathrm{C}$ ), the spherulites of both constituents were observed by POM. In particular, both components simultaneously crystallized at

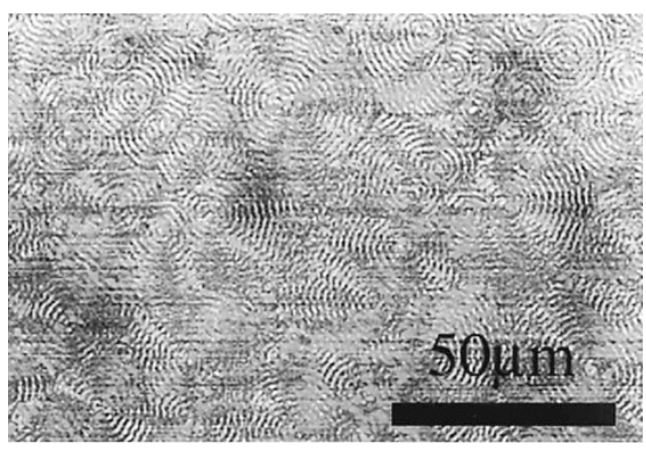

Figure 3. Typical POM image in regime i. $\phi_{\mathrm{PLLA}}=0.4, T_{\mathrm{c}}=$ $50^{\circ} \mathrm{C}$, the crystallization time $t=1 \mathrm{~min} 30 \mathrm{sec}$.

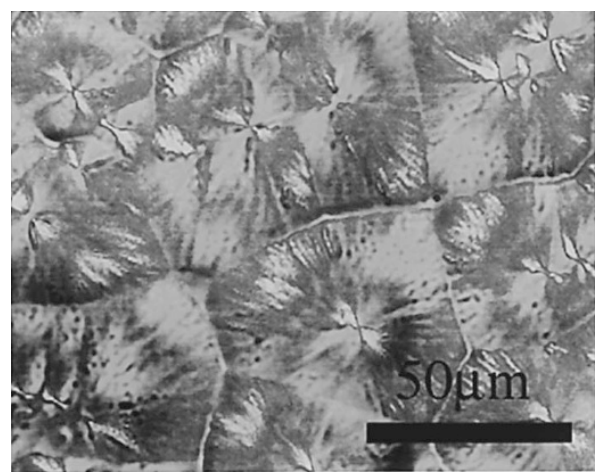

Figure 4. Typical POM image in regime iv. $\phi_{\mathrm{PLLA}}=0.6, T_{\mathrm{c}}=$ $85^{\circ} \mathrm{C}, t=8 \mathrm{~min}$.
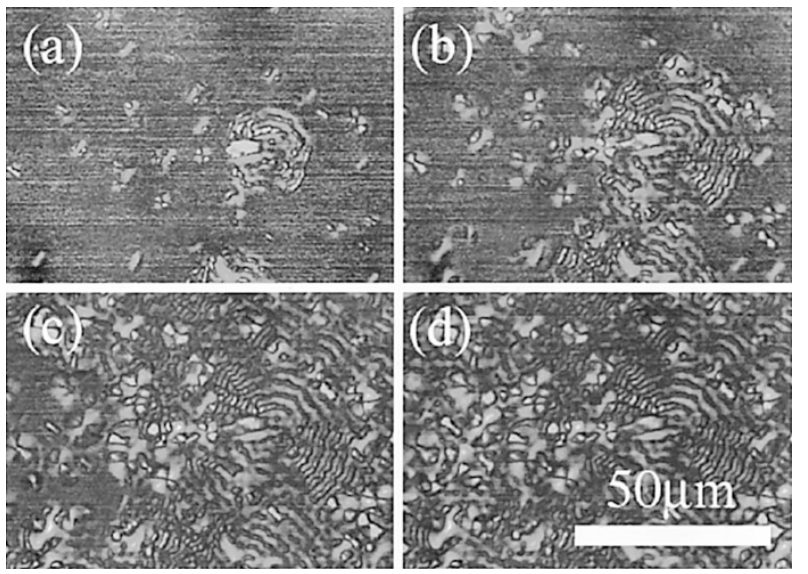

Figure 5. The spherulitic growth of a blend $\phi_{\mathrm{PLLA}}=0.4$ and $T_{\mathrm{c}}=65^{\circ} \mathrm{C}$ (regime ii). The crystallization times were (a) $1 \mathrm{~min} 30$ sec, (b) $2 \min 30 \mathrm{sec}$, (c) $3 \mathrm{~min} 30 \mathrm{sec}$, and (d) $4 \mathrm{~min} 30 \mathrm{sec}$.

$0.1 \lesssim \phi_{\text {PLLA }} \lesssim 0.6$ and $60^{\circ} \mathrm{C} \lesssim T_{\mathrm{c}} \lesssim 70^{\circ} \mathrm{C}$ (regime ii). This is because the values of $G$ of PEC and PLLA were almost the same in this regime. Figure 5 shows typical spherulitic growth in regime ii. The spherulites with extinction rings are PEC spherulites, and the slightly darker spherulites without rings are PLLA spherulites. The crystallization process is as follows. The PEC and PLLA spherulites nucleated almost simultaneously. After a fast-growing PEC spherulite contacted with a slowly-growing PLLA spherulite, the brighter birefringent patterns of PEC continued to spread inside the 


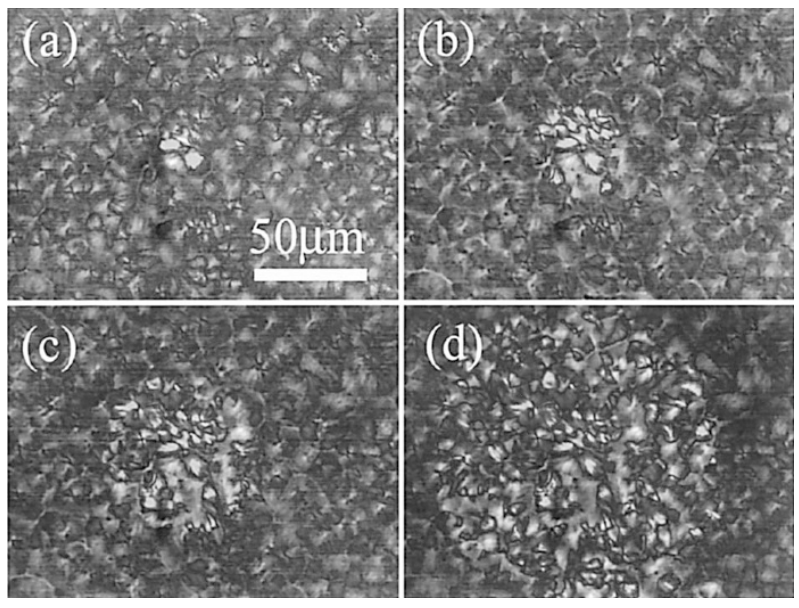

Figure 6. The spherulitic growth in a blend $\phi_{\mathrm{PLLA}}=0.4$ and $T_{\mathrm{c}}=85^{\circ} \mathrm{C}$ (regime iii). The crystallization times were (a) $13 \mathrm{~min}$ $30 \mathrm{sec}$, (b) $21 \mathrm{~min}$, (c) $30 \mathrm{~min}$, and (d) $50 \mathrm{~min} 30 \mathrm{sec}$.

PLLA spherulite although the extinction rings disappeared in this area. The PEC spherulites continued to grow until they filled the whole space, while PLLA spherulites stopped growing after the contact with PEC or other PLLA spherulites.

For $0.2 \lesssim \phi_{\text {PLLA }} \lesssim 0.6$ and $70^{\circ} \mathrm{C} \lesssim T_{\mathrm{c}} \lesssim 90^{\circ} \mathrm{C}$, the growth of the two components was also observed though the crystallization behavior was different from that in regime ii (regime iii). The values of $G$ were larger and the nucleation rate was larger for PLLA than for PEC in regime iii. PLLA therefore crystallized first and filled the whole space before PEC started to crystallize as shown in Figure 6. A brighter PEC spherulite started to crystallize in the matrix of PLLA spherulites. The bright pattern of PEC continued to grow until it collided with a neighboring bright pattern of another PEC spherulite.

Figure 7 summarizes the crystallization behavior of the PEC/PLLA blends. The crystallization process was classified into four regimes according to $\phi_{\text {PLLA }}$ and $T_{\mathrm{c}}$. The regime $\mathrm{i}$ was observed under the conditions of $\phi_{\text {PLLA }} \lesssim 0.4$ and $T_{\mathrm{c}} \lesssim 60^{\circ} \mathrm{C}$. In this case, the PEC spherulites started to crystallize and filled the whole space. In contrast for $0.2 \lesssim \phi_{\text {PLLA }}$ and $90^{\circ} \mathrm{C} \lesssim T_{\mathrm{c}}$, the PLLA spherulites were observed (regime iv). Under the intermediate conditions $\left(0.1 \lesssim \phi_{\text {PLLA }} \lesssim 0.6\right.$ and $60^{\circ} \mathrm{C}$ $\lesssim T_{\mathrm{c}} \lesssim 90^{\circ} \mathrm{C}$ ), both PEC and PLLA spherulites were observed (regimes ii and iii).

We now discuss the possibility of penetration of the spherulites in regimes ii and iii. Figure 8 shows the models of the change in birefringence of PEC and PLLA spherulites when they crystallize simultaneously. If they were superposed on each other, the retardation would increase where they have the same birefringence and would decrease where they have the opposite birefringence. For example, when a PLLA

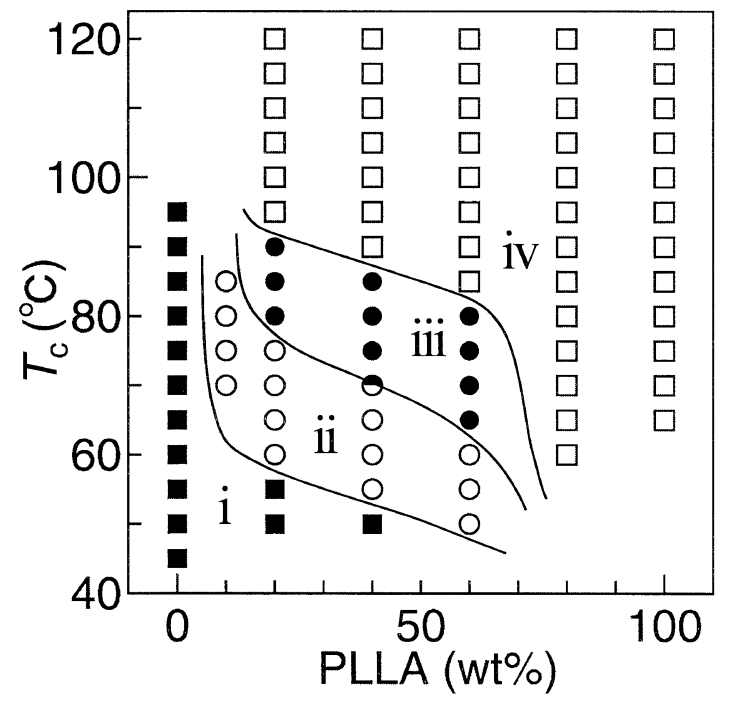

Figure 7. The crystallization patterns observed by POM. Only PEC spherulites crystallized (regime i). $\bigcirc$ : Both components crystallized simultaneously (regime ii). $\mathbf{0}$ : PEC spherulites started to crystallize after PLLA spherulites completely filled the whole observation area (regime iii). $\square$ : Only PLLA spherulite crystallized (regime iv).

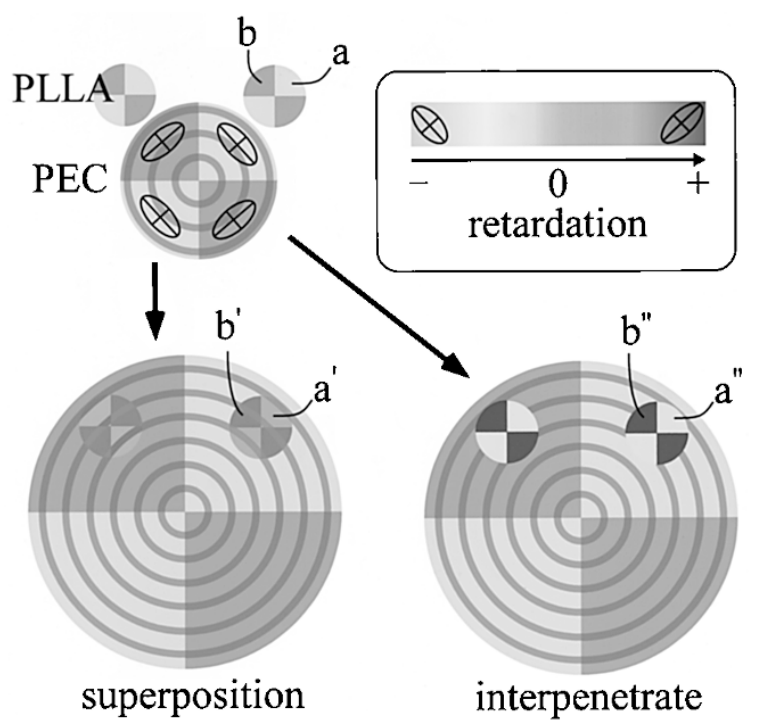

Figure 8. The models of the change in color of PEC and PLLA spherulites observed by POM. The figure insetted shows the correspondence of the retardation to the color of the transmitted light. The birefringent ellipsoids are also shown.

spherulite is superposed on the right shoulder of a PEC spherulite, the right shoulder of the PLLA spherulite (Figure 8a) have the same birefringence so that the retardation would increase (Figure $8 \mathrm{a}^{\prime}$ ). On the contrary, the left shoulder of the PLLA spherulite (Figure $8 \mathrm{~b}$ ) has the opposite birefringence so that the retardation would decrease (Figure $8 b^{\prime}$ ). When the retardation changes, the wavelength of the transmitted light would change with a test plate. Further more, PEC spherulites would show extinction rings in the areas of PLLA spherulites. However, the experimental results showed the increase 
in light intensity of PLLA spherulites, the unchanged color of the spherulites, and no extinction rings of PEC in the area of PLLA spherulites. These results indicate that PEC and PLLA spherulites are not superposed on each other. In PEC/PLLA blends, both components show negative birefringence, that is, their birefringent ellipsoids are considered to be oriented to the same direction against lamellae. When one component crystallizes along the lamellae of the other, the birefringence is expected to keep negative (Figure $8 a^{\prime \prime}, 8 b^{\prime \prime}$ ). This situation is analogous to that of PBSU/PVDCVC blends, where PBSU lamellae crystallized in PVDCVC spherulites along the PVDCVC lamellae and PVDCVC spherulites stopped growing. ${ }^{1-14}$ This analogy indicates the possibility of penetration of PEC into PLLA spherulites along the lamellae or fibrils of PLLA.

\section{CONCLUSION}

The single $T_{\mathrm{g}}$ of the DSC curves that changed according to the Fox equation indicated miscibility of PEC/PLLA blends. For $0.1 \lesssim \phi_{\text {PLLA }} \lesssim 0.6$ and $60^{\circ} \mathrm{C} \lesssim T_{\mathrm{c}} \lesssim 90^{\circ} \mathrm{C}$, spherulites of both components were observed, and for $0.1 \lesssim \phi_{\text {PLLA }} \lesssim 0.6$ and $60^{\circ} \mathrm{C}$ $\lesssim T_{\mathrm{c}} \lesssim 70^{\circ} \mathrm{C}$, they crystallized simultaneously. Under such conditions, PEC spherulites continued to grow after the collision with PLLA spherulites, while PLLA spherulites stopped growing. The change in birefringence of PLLA spherulites indicated the penetration of PEC lamellae into PLLA spherulites. More detailed in- formation about $G$ and nucleation rate will be presented elsewhere. Also, we are working on the direct observation of interpenetrated spherulites in higher resolution by AFM.

\section{REFERENCES}

1. M. Aubin, Y. Bedard, M. F. Morrissette, and R. E. Prud'homme, J. Polym. Sci., Part B: Polym. Phys., 21, 233 (1983).

2. H. Zhang and R. E. Prud'homme, J. Polym. Sci., Part B: Polym. Phys., 24, 233 (1987).

3. M. Avella and E. Martuscelli, Polymer, 29, 1731 (1988).

4. E. Blumm and A. J. Owen, Polymer, 36, 4077 (1995).

5. J. P. Penning and R. St. John Manley, Macromolecules, 29, 77 (1996).

6. J. P. Penning and R. St. John Manley, Macromolecules, 29, 84 (1996).

7. K. Fujita and T. Kyu, Macromolecules, 29, 91 (1996).

8. L.-Z. Liu, B. Chu, J. P. Penning, and R. St. John Manley, Macromolecules, 30, 4398 (1997).

9. J.-C. Lee, H. Tazawa, T. Ikehara, and T. Nishi, J. Polym. Sci., Part B: Polym. Phys., 352645 (1997).

10. J.-C. Lee, H. Tazawa, T. Ikehara, and T. Nishi, Polym. J., 30, 327 (1998).

11. J.-C. Lee, H. Tazawa, T. Ikehara, and T. Nishi, Polym. J., 30, 780 (1998).

12. T. Ikehara and T. Nishi, Polym. J., 32, 683 (2000).

13. Y. Terada, T. Ikehara, and T. Nishi, Polym. J., 32, 900 (2000).

14. S. Hirano, Y. Terada, T. Ikehara, and T. Nishi, Polym. J., 33, 371 (2001).

15. T. J. Fox, Bull. Am. Phys. Soc., 1, 123 (1956). 